\title{
The COVID-19 Crisis, Indigenous Peoples, and International Law
}

\author{
A Vulnerability Perspective
}

\author{
Malgosia Fitzmaurice
}

Contemplating our shared vulnerability it becomes apparent that human beings need each other, and that we must structure our institutions in response to this fundamental human reality. ${ }^{1}$

Research undertaken globally in the wake of the COVID-19 pandemic indicates that resilience gaps that are already the status quo in respect of indigenous peoples are situating numerous communities at imminent risk of disaster. This short essay presents a brief risk profile analysis carried out by reference to Martha Fineman's theory of vulnerability. Extensive vulnerability manifestations are identified according to different sets of relationships, featuring significant differential barriers for access to goods and services, which place indigenous peoples in a situation of alarming exposure to the impacts of COVID-19. This author argues that awareness of these relationships and obstacles can and should guide State responses, and highlight the role of law as a necessary tool for enabling and maintaining much needed, appropriately targeted resilience work.

One of the principal callings of international law is the establishment of frameworks for State conduct as pathways through which to address shared challenges. Yet, most scholarship involving the transcendent crises of our time, from human rights to the erosion of the Earth's natural resources and environment, illuminates how the solidarity demands placed on States by such crises often expose the limitations of international law. The current COVID-19

1 Martha Fineman, 'The Vulnerable Subject: Anchoring Equality in the Human Condition' (2008) 2o(1) Yale Journal of Law and Feminism 1, 12. 
pandemic evidences these limitations once more, both in respect of the search for effective international responsibility, and in meeting the urgent, broader, more complex protection and support needs of vulnerable populations. Nevertheless, it is difficult to imagine the articulation of successful international responses to stem the crisis without apposite international frameworks. In this paper, appropriate State responses for indigenous peoples are explored , as they face the CoviD-19 pandemic in a marked position of comparative disadvantage, with exposure to the disease situating them in danger of increased mortality. ${ }^{2}$

COVID-19 is having a particularly nefarious impact in regions predominantly inhabited by indigenous populations where health systems were already fragile, and may now have collapsed leaving vulnerable groups in an unprecedented state of exposure and risk. Diverse but often compounding factors are relevant in driving this undesirable outcome for many indigenous communities. Biological characteristics can play a part, ${ }^{3}$ but they are not always the main factor, with different cultural, political, and socio-economic causes also driving this alarming trend. As this author has previously argued, the personal and socio-economic characteristics of many human communities, including indigenous groups, merit the application of Martha Fineman's theory of vulnerability. ${ }^{4}$ In Fineman's own words: 'The theory is based on a descriptive account of the human condition as one of universal and continuous vulnerability'. She adds further that: 'The potential normative implications of the theory are found in the assertion that State policy and law should be responsive to human vulnerability. However, the call for a responsive State does not dictate the form responses should take, only that they reflect the reality of human vulnerability. ${ }^{5}$ The key implication of the theory is that formal equality, whilst at times an appropriate response, as in the case of for example voting rights, is in many other aspects of life not achievable as an aspirational objective, and may even result in manifest unfairness in cases where individuals are differently situated across diverse societal contexts.

2 Tamara Power and others, 'COVID-19 and Indigenous Peoples; An imperative for action' (2020) 29 Journal of Clinical Nursing 2737.

3 Tony Kirby, 'Evidence mounts on the disproportionate effect of COVID-19 on ethnic minorities' (2020) 8(6) Lancet Respiratory Medicine 547.

4 See Martha Fineman, 'Vulnerability and Inevitable Inequality' (2017) 4(3) Oslo Law Review 133 .

5 ibid 133 . 
From Fineman's perspective, vulnerability is a constant for all peoples, but one that has different manifestations across society resulting in varying degrees of social dependency across the population spectrum. Fineman illustrates the theory in the context of the family, where gender inequality has historically been a feature, relying on the example of reform efforts premised on formal equality in spite of less than equal outcomes. Fineman suggests that institutional responsibility may imply unequal treatment in favour of persons who are either inevitably dependent (such as children, the elderly or those with a severe disability), or those whose vulnerability is not inevitable, but who are less resilient due to their position and role in society (for example, carers or socially excluded or structurally subordinated groups). Hence, vulnerability responsive laws and policies are intended to account and compensate for unequal needs across different social contexts. ${ }^{6}$

Under the vulnerability lens, resilience is thus not innate to the human being, but rather conferred by inclusion in the social and institutional relationships, many of which will be underpinned by public institutions, and defined and maintained by law. This approach has the effect of relieving the onus that the liberal focus places on individuals to transcend the specific vulnerability contexts in which they are situated. Physical, social, cultural, and material wellbeing and development are not innate to the human being, but dependent on inclusion and maintenance within the social, economic, and institutional relationships that promote resilience. Social identities that may or may not reflect the individual characteristics of people may promote inclusion or exclusion from such networks, processes, and relationships. Some of those social identities may change through life (e.g. infant, adult, elderly) but others are stable and can influence an individual's options throughout his or her lifetime. The State, through its public organs and agencies, has the responsibility of modulating and redefining such relationships for the facilitation of resilience, taking care that they do not perpetuate egregious inequality.

This approach has considerable implications for the development and reform of law and policy, including in the international context. As Fineman explains: "[t]he abstract and inevitably contested legal principles often referred to in human rights literature, such as equality, liberty, and dignity, are not the measure for this inquiry, however'. Nevertheless, individuals and

6 Fineman (n 4) 138-141. 
their characteristics, both in respect of permanent vulnerability features as well as life stages, are as relevant to the analysis as the set of social institutions and relationships to which they pertain. Particularly important is the need to identify the differential relationships that characterize indigenous communities. Sets include institutions and relationships that either favour or impede sanitation, education, employment, health, connectivity - to name a few. The first task of vulnerability informed policy is monitoring these institutions and relationships so that the relevant State organs can perceive situations of vulnerability, and respond to them in order to promote resilience. According to Fineman, such resilience is strengthened via access to categories of goods and services, as follows: Human capital goods such as education and training, and social resources such as family, community including ethnicity, and political networks. Fundamental, of course, are physical goods, such as food, sanitation, savings, housing, and transport. Environmental resources refer to the lived environment and its services and threats, whereas existential resources refer to beliefs, including religion but also culture, allowing an understanding of the world and life events. These are the resilience categories through which the State can assess and address the vulnerability of fragile human communities.

, I will commence my analysis with an enquiry on access by indigenous communities to the institutions or processes whereby the allocation of resources by the State takes place in order to outline a broad vulnerability pathway. The analysis intends to assist in the definition of a strategy able to alleviate the vulnerability status of indigenous communities in the specific context of the pandemic. The point of departure is to identify whether the structural features of exclusion and deprivation that affect indigenous communities relate to any of the above categories.

Now the physical goods and services category will be addressed. Abundant evidence indicates that, throughout the world, many indigenous populations have been and continue to be excluded, remaining chronically disengaged from the provision of basic public services, such as access to clean water and other necessary infrastructure. Women, children, and people living with disabilities suffer particularly grievously from the effects of such marginalisation. The impact of the pandemic means that these groups are likely to be facing even more precarity, and be disproportionally affected by severe risks. The consequences of the pandemic are likely to exacerbate already significant levels of 
insecurity in matters of sustenance and health. ${ }^{7}$ Elevated rates of transmission are also due to the conditions of poverty in which many of these communities are forced to live. Poor housing quality and lack of sanitation, crowding, and precarious infrastructures result in a diminished capacity for adaptability when confronted with the social and economic restrictions that are being adopted as mitigating responses to COVID-19.

$\mathrm{t}$ I will address now human goods category, which is closely connected to the existential sphere in a significant way, as information and cultural acumen enables individuals to process and understand the significance of events, and their implications for themselves and their families and communities. Communication limitations and ensuing exclusion thus have the potential to unfairly place entire peoples in situations that limit or prevent timely responses against critical events, such as the onset of a pandemic. Cultural difference and marginalisation play a significant part in the vulnerability of indigenous groups, and communication strategies can and often do fail to be incorporated into community systems. This can result in reduced opportunities for understanding and implementing adequate responses, potentially with disastrous consequences. The lack of visibility of some extremely marginalized groups can also be a factor in causing and/or compounding those barriers, resulting in increased exposure and ultimate defenselessness against coviD-19. These issues are not unique to particular geographic areas, and similar trends have been observed across different continents. ${ }^{8}$

Next, I turn to the overview of the category of environmental goods and services. Many indigenous communities live in rural areas, relying on the harvesting of wild species of plants and animals for nutrition as well as medicine. This context is also proving to be a risk factor, particularly at a time when the pandemic is accentuating the impact of climate change on food productivity, compounding pressures on traditional custody chains, and eroding already fragile life and community supporting practices and structures. ${ }^{9}$ In this regard,

7 'COVID-19 and the world of work: A focus on indigenous and tribal peoples' (International Labour Organisation 2020) <https://www.ilo.org/wcmsp5/groups/public/ - -dgreports/ - dcomm/documents/publication/wcms_746893.pdf> accessed 25 September 2020.

8 See, for example, Melissa McLeod and others, 'COVID-19: we must not forget about indigenous health and equity' (2020) 44(4) Australian and New Zealand Journal of Public Health 253; Sergio Meneses-Navarro and others, 'The Challenges facing indigenous communities in Latin America as they confront the COVID-19 pandemic' (2020) 19 International Journal for Equity in Health 63; Lucas Ferrante and Philip Fearnside, 'Protect Indigenous peoples from COVID-19' (2020) 368 (6488) Science Magazine 251.

9 Carol Zavaleta-Cortijo and others, 'Climate change and COVID-19: reinforcing Indigenous food systems' (2O20) 4(9) The Lancet $\mathrm{E}_{3} 81-382$. 
the particularly acute situation of crisis that is being experienced by indigenous groups can, at least in part, be traceable to the fragility of the natural environments in which they live, the often stressed and unprotected ecosystems that sustain their livelihoods, and their dependency on the essential services emanating from them.

A pertinent example may be seen in the impact of the coronavirus on smallscale fisheries, including capture, processing and support activities along the production and custody chain, all of which supports the food and work security of numerous subsistence communities globally, including indigenous communities. Despite the emergence of limited governmental, grassroots, and other initiatives to counteract the effect of CoviD-19 on small-scale fisheries and fishing communities across the world, ${ }^{10}$ high dependency on small-scale fisheries for food and work makes this a high-risk area. Beyond subsistence, the concentration of transactions in a relatively small number of key markets means impacts have the potential to be devastating for communities that depend on such trade, highlighting an acute need for adaptation and resilience mechanisms to provide viable alternatives in the event of closures. ${ }^{11}$ To put risks in context with some figures, it is estimated that circa $5^{2}$ million people are employed in small-scale capture fisheries around the world, with the livelihoods of an even higher number being supported by the provision of services provided on land to the custody chain of small-scale fishery products. ${ }^{12}$ In addition, the nature of fisheries production means that product survival is dependent on critical services such as the provision of ice and other cold or freezing facilities that may not be available during the pandemic. Further, some aspects of the custody chain present enhanced risk of contagion, due to exposure to crowded working conditions on board and on land, ${ }^{13}$ and other possible contributory factors such as the handling of raw produce.

Of course, environmental contexts extend beyond rural and coastal settings. Other production scenarios also present enhanced vulnerabilities to CoviD-19, particularly those in which informal and precarious work is significant. These

\footnotetext{
10 'Information on COVID-19 and small-scale fisheries' (Food and Agriculture Organization of the United Nations 2020) <http://www.fao.org/3/ca8959en/ca8959en.pdf> accessed 25 September 2020 .

11 Christopher Knight and others, 'COVID-19 reveals vulnerability of small-scale fisheries to global market systems' (2O2O) 4(6) Lancet Planet Health E219.

12 Hilary Smith and Xavier Basurto, 'Defining Small-Scale Fisheries and Examining the Role of Science in Shaping Perceptions of Who and What Counts: A Systematic Review' (2019) 6 Frontiers in Marine Science 236.

13 Nathan Bennet and others, 'The COVID-19 Pandemic, Small-Scale Fisheries and Coastal Fishing Communities' (2020) 48(4) Costal Management 336.
} 
contexts include other food production sectors, as well as urban settings in which domestic work, hospitality, transport, manufacturing, and construction services are abundant. According to the International Labour Organization (ILO), indigenous peoples are significantly over-represented in these work contexts. The ILO's work focusing on indigenous and tribal people indicates that long-standing marginalisation factors can explain their particular exposures to covid-19. These vulnerabilities cannot be accurately described as exceptional, as indigenous and tribal populations constitute circa $6 \%$ of the world population. ${ }^{14}$

The social category will be now analysed, noting that it extends into the political sphere. According to the ILO, public institutions dedicated to the situation of indigenous people and their development and integration into public life, whilst present and in development in some countries, are still absent in many others. The ILo has called for the creation and strengthening of such institutions, in order to combat the systematic exclusion of indigenous groups in many countries. ${ }^{15}$ In the context of the pandemic, porosity across these categories is likely to compound vulnerability. For example, the absence of dedicated institutions is likely to make access to information regarding health adaptations more difficult, thus also highlighting a human capital need.

Understandings of vulnerability and its causes in respect of the plight of indigenous peoples have extended beyond material concerns, to include a historical lack of rights to access resources and opportunities in the context of modern societies. ${ }^{16}$ The most comprehensive international legal instrument concerning the protection of indigenous communities is the 2007 Declaration on the Rights of Indigenous Peoples, adopted by the United Nations (UN) General Assembly. This document covers extensive collective rights of indigenous people, as are considered indispensable not only for their survival, but also for their well-being, culture, resources, and self-determination. Yet, indigenous communities still face immense obstacles for the recognition and substantiation of their rights and dignity, which in effect places them in a situation of comparative disadvantage vis-à-vis other communities. In 2019, the UN reported that, although indigenous rights are recognised internationally, more

\footnotetext{
$14 \quad \operatorname{ILO}\left(\mathrm{n}_{7}\right) 6$.

15 ibid.

16 Amartya Sen, 'Sobre Conceptos y Medidas de Pobreza' (1992) 42(4) Comercio Exterior 1.
} 
work needs to be done to ensure their implementation, and to facilitate their enforcement, so that they are safeguarded and respected by public and private entities alike, and to prevent abuses. ${ }^{17}$ In many countries, this lack of rights has led to pervasive and long-standing inequality, preventing access to services that are essential to human health, basic education and development. As a consequence of these and other compounding causes, indigenous communities suffer from a higher rate of morbidity and mortality in comparison to other groups, and are now facing the effects of the pandemic from a starting point of long-standing deprivation and fragility. ${ }^{18}$

There is little dispute that the collective and individual frailty of physical, economic, and communicative resources, and lower health baseline, places indigenous individuals and communities in an alarming position in the face of the CoviD-19 pandemic. There is a need to incorporate this urgency into State responses to the pandemic, to substantiate the rights and meet the needs of indigenous communities across the physical, human, socio-political, environmental, and existential spheres. This approach can inform the focus, dissemination, substantive content, and granularity of policies. This approach is consistent with the urgency, coordination and sharing of resources that is required in a global pandemic, and fully in line with the objectives of the UN Declaration on the Rights of Indigenous Peoples. Nevertheless, given that domestic implementation obstacles have been a persistent factor in impermissible and persistent discrimination, resulting in indigenous exclusion and abandonment, the focus from a legal perspective should specifically be placed on the responsibility of the State, as authority with the furthest reaching powers and duties. Even though many countries have taken measures against poverty, it is noteworthy that they have seldom been successful in addressing the causal conditions that keep indigenous populations in a particularly high vulnerability status. Nevertheless, the States that have implemented the 1989 Indigenous and Tribal Peoples Convention are likely to possess a more solid point of departure, as implementation will result in economic assessments likely to cover some of the categories described above.

17 United Nations Department of Economic and Social Affairs, 'State of the World's Indigenous Peoples: Implementing the United Nations Declaration on The Rights of Indigenous Peoples' UN Doc ST/ESA/371 (sowIP vol IV 2019) 69.

18 United Nations Department of Economic and Social Affairs, 'State of the World's Indigenous Peoples: Indigenous Peoples' Access to Health Services' (sowiP vol II 2016) 7 . 


\section{Concluding Observations}

Through the prism of vulnerability, structural safeguarding needs are revealed as foundations for action, highlighting the necessity of embracing resilience as a guiding objective for the development of international law. CoviD-19 has increased the need for urgency in protecting and supporting vulnerable individuals and communities. The vulnerability framework can guide law making efforts and the establishment of policies to effectively provide relief where it is most needed. The plight of indigenous communities, where enduring conditions of exclusion and marginalisation have already been documented, should be prioritised in order to eliminate egregious systemic inequality. Implicit in the brief analysis presented in this paper is a call for swift State responsiveness, and for action to support the facilitation of resilience to these communities. In the short term, prioritisation should be given to the protection of the physical systems that support vital needs, especially food security, and adequate sanitation and health preservation measures. Ensuring the human services necessary for the establishment of effective and vital communication strategies will need to be incorporated into health safeguarding strategies. Towards the medium term, these urgent objectives should be reinforced with mechanisms to protect indigenous and traditional knowledge sources with regard to food availability and medicine effectiveness, availability, and accessibility. Understanding the impact, viability, and protection of extraction methods from already fragile natural environments will be key, as is the implementation of work safety and security policies. Much of this work implies looking beyond immediate emergency actions, and reaching further towards increased collective autonomy, and meaningful inclusion of indigenous peoples in sociographic and political processes. ${ }^{19}$ This task cannot be accomplished without a commitment to resilience, and without appropriate legal tools capable of ensuring that it is achieved, and maintained. The shock of COVID-19 has been shattering, and there is a clear need to secure as well as redefine the structures that contain, protect, and support people and communities. This time, work should commence with the most vulnerable.

19 For further insight, see Per Axelsson, and Peter Sköld, 'Indigenous Populations and Vulnerability: Characterizing Vulnerability in a Sami Context' (2006) I(III) Annales de Demographie Historique 115 . 